\title{
Near BPS Wilson Loop in $\beta$-deformed Theories
}

\author{
Chong-Sun Chu, Dimitrios Giataganas \\ Department of Mathematical Sciences, University of Durham, Durham, DH1 3LE, UK \\ chong-sun.chu@durham.ac.uk, dimitrios.giataganas@durham.ac.uk
}

\begin{abstract}
We propose a definition of the Wilson loop operator in the $\mathcal{N}=1 \beta$-deformed supersymmetric Yang-Mills theory. Although the operator is not BPS, it has a finite expectation value at least up to order $\left(g^{2} N\right)^{2}$. This does not happen generally for a generic non-BPS Wilson loop whose expectation value is UV divergent. For this reason we call this a near-BPS Wilson loop and conjecture that its exact expectation value is finite. We derive the general form of the boundary condition satisfied by the dual string worldsheet and find that it is deformed. Finiteness of the expectation value of the Wilson loop, together with some rather remarkable properties of the Lunin-Maldacena metric and the $B$-field, fixes the boundary condition to be one which is characterized by the vielbein of the deformed supergravity metric. The Wilson loop operators provide natural candidates as dual descriptions to some of the existing D-brane configurations in the Lunin-Maldacena background. We also construct the string dual configuration for a near-1/4 BPS circular Wilson loop operator. The string lies on a deformed three-sphere instead of a two-sphere as in the undeformed case. The expectation value of the Wilson loop operator is computed using the AdS/CFT correspondence and is found to be independent of the deformation. We conjecture that the exact expectation value of the Wilson loop is given by the same matrix model as in the undeformed case.
\end{abstract}




\section{Contents}

1. Introduction $\quad 0$

2. The Lunin-Maldacena Background 3

2.1 Properties of the deformed metric and $B$-field 4

3. Near-BPS Wilson Loop and Twisted Boundary Condition 6

3.1 Form of the Wilson loop operator 6

3.2 Deformed boundary conditions 7

3.3 Legendre transformation and boundary contribution 9

4. Near-1/4 BPS Wilson Loop $\quad 10$

A. Wilson loop from $U(N+1) \rightarrow U(N) \times U(1)$ breaking

B. The deformed metric in the Cartesian coordinate system 17

C. Derivation of the Hamilton-Jacobi Equation $\quad 19$

D. Cancellation of UV divergences up to order $\left(g^{2} N\right)^{2} \quad 20$

\section{Introduction}

The AdS/CFT correspondence states the equivalence of string theory on $A d S_{5} \times S^{5}$ to the $\mathcal{N}=4$ supersymmetric Yang-Mills [1-4]. According to this correspondence, there exists a map between gauge invariant operators in the field theory and states in the string theory. The correspondence is well understood for the case of half BPS local operators where the dual string states are Dbranes in the bulk $[5,6]$. The Wilson loop operator is another important class of gauge invariant observable which is non-local. The Wilson loop operator in the Euclidean $\mathcal{N}=4$ SYM theory is given by [7]

$$
W_{R}[C]=\frac{1}{N} \operatorname{Tr}_{R} P \exp \left(\oint_{C} d \tau\left(i A_{\mu} \dot{x}^{\mu}+\varphi_{i} \dot{y}^{i}\right)\right),
$$

where $A_{\mu}$ are the gauge fields and $\varphi_{i}$ are the six real scalars. The loop $C$ is parametrized by the variables $\left(x^{\mu}(\tau), y^{i}(\tau)\right)$, where $\left(x^{\mu}(\tau)\right)$ determines the actual loop in four dimensions, and $\left(y^{i}(\tau)\right)$ can be thought of as the extra six coordinates of the ten-dimensional $\mathcal{N}=1$ super Yang-Mills theory, of which theory is the dimensionally reduced version. $R$ is the representation of the 
gauge group $G$. In this paper we will be interested in the case $G=U(N)$. In (1.1), the coupling to the gauge fields and the scalar fields is controlled by $\dot{x}^{\mu}$ and $\dot{y}^{i}$. In particular, Wilson loop operator satisfying the constraint

$$
\dot{x}^{2}=\dot{y}^{2}
$$

is locally BPS. Moreover it has a finite expectation value.

In the AdS/CFT correspondence, BPS Wilson loop operators in the fundamental representation is dual to a fundamental string worldsheet ending on the $A d S_{5}$ boundary $[7,8]$. Recently, it has been realized that Wilson loop in the symmetric or antisymmetric representation can be described in terms of a single D3-brane or D5-brane with worldvolume RR flux. See [9-15] for the 1/2 BPS case and [16] for the D3-brane dual for 1/4 BPS Wilson in symmetric representation. More generally, it has been shown in $[11,14]$ that half BPS Wilson loop operators in general higher rank representations can be described in terms of a certain array of D3 branes or D5-branes. Analogous to the approach of [17], the supergravity description for certain half BPS Wilson loop has also been obtained [18-21].

The goal of this paper is to try to extend some of these results to theories with less supersymmetries. We will consider an $\mathcal{N}=1$ supersymmetric gauge theory obtained by a marginal $\beta$-deformation of the $\mathcal{N}=4 \mathrm{SYM}$. The theory is described by the superpotential

$$
i h \operatorname{Tr}\left(e^{i \pi \beta} \Phi_{1} \Phi_{2} \Phi_{3}-e^{-i \pi \beta} \Phi_{1} \Phi_{3} \Phi_{2}\right),
$$

where $\Phi_{i}$ are the three $\mathcal{N}=1$ chiral superfields. The theory is conformal provided a condition on the parameters $h, \beta$ and the gauge coupling $\tau$ is satisfied. The resulting theory preserves $\mathcal{N}=1$ superconformal symmetry and has a global $U(1) \times U(1)$ symmetry

$$
\begin{array}{ll}
U(1)_{1}: & \left(\Phi_{1}, \Phi_{2}, \Phi_{3}\right) \rightarrow\left(\Phi_{1}, e^{i \delta_{1}} \Phi_{2}, e^{-i \delta_{1}} \Phi_{3}\right) \\
U(1)_{2}: & \left(\Phi_{1}, \Phi_{2}, \Phi_{3}\right) \rightarrow\left(e^{-i \delta_{2}} \Phi_{1}, e^{i \delta_{2}} \Phi_{2}, \Phi_{3}\right) .
\end{array}
$$

The $U(1)_{R}$ symmetry acts as

$$
U(1)_{R}: \quad\left(\Phi_{1}, \Phi_{2}, \Phi_{3}\right) \rightarrow e^{i \delta}\left(\Phi_{1}, \Phi_{2}, \Phi_{3}\right)
$$

under a rotation $\theta \rightarrow e^{3 i \delta / 2} \theta$. All together, the $\mathcal{N}=1 \beta$-deformed SYM theory is invariant under a $U(1)^{3}$ symmetry. It's action on the scalar components is

$$
\Phi_{k} \rightarrow e^{i \delta_{k}} \Phi_{k}, \quad \text { for arbitrary constants } \delta_{k}, \quad(k=1,2,3) .
$$

Here we have used the same notation $\Phi_{k}$ to denote both the lowest component of the superfield as well as the superfield itself.

The supergravity dual of the $\beta$-deformed SYM was found by Lunin and Maldacena in [26]. The Lunin-Maldacena background can be obtained from the $A d S_{5} \times S^{5}$ via a series of T-duality transformation, shift and T-duality transformation acting on the five-sphere (S-duality is also needed if $\beta$ is complex). We will look at the real $\beta$ case. The supergravity description is valid in the limit of small curvature $R=\left(4 \pi g_{s} N\right)^{1 / 4} \gg 1$ and

$$
R \beta \ll 1,
$$


with

$$
R^{2} \beta:=\hat{\gamma} \quad \text { fixed }
$$

Aspects of the supergravity duals of Wilson loops in the $\beta$-deformed SYM theory has been studied before $[22,23]^{1}$. However the form of the field theory operators that are in dual with the supergravity configurations has not been identified. We note that the Wilson loop operator (1.1), (1.2) is non-BPS since the gauge bosons and the scalars are in different $\mathcal{N}=1$ supersymmetry multiplets and so their supersymmetry variations cannot cancel out each other. Conformal supersymmetry also does not mix these multiplets. ${ }^{2}$ One can check that even by allowing general fermion couping, it is not possible to construct a supersymmetric Wilson loop. It thus appears impossible to construct a Wilson loop operator which respects some of the $\mathcal{N}=1$ superconformal symmetries of the $\beta$-deformed SYM.

In this paper we point out that although the Wilson loop operator (1.1), (1.2) is non-BPS ${ }^{3}$, it shares a distinguished property of the locally BPS Wilson loop operator in the $\mathcal{N}=4$ theory namely, it has a finite vev. This is not true for a generic non-BPS Wilson loop. To distinguish it from a generic non BPS loop, we call the operator (1.1), (1.2) a near BPS Wilson loop operator. An analogous example is the BMN operator in the $\mathcal{N}=4 \mathrm{SYM}$ theory. The BMN operator is not a BPS operator, but it has a finite anomalous dimensions in a particular double scaling limit [25]. This operator is very interesting and have been studied extensively. We stress that the near BPS Wilson loop operator is not a deformation of a BPS one. The use of "near" is to emphasis that although it is not BPS, but it has finite expectation value just as a BPS Wilson loop operator in the $\mathcal{N}=4$ theory does.

We propose that dual operators for the D-brane configurations in [23] are given by the near $B P S$ Wilson loop operators (1.1), (1.2) whose path is a circle in the $x$-space and a point in the transverse space. When $\beta \approx 0$, an approximate half of the associated $\mathcal{N}=4$ supersymmetry is preserved. And one may call this Wilson loop operator near-half BPS. We also consider the near-1/4 BPS case and construct the dual microscopic string description. The Wilson loop's expectation value is computed using the AdS/CFT correspondence and, as expected, it is finite. Unlike the near-1/2 BPS Wilson loops where the authors find that precisely the same undeformed ansatz has to be taken to construct the desired dual D-branes configurations, here we find that one has to employ a modified ansatz to construct the dual string minimal surface.

The paper organized as follows. In section 2, we review the Lunin-Maldacena background in its original form where the deformed sphere metric is written in the angular coordinate system.

\footnotetext{
${ }^{1}$ The construction of [23] utilizes some interesting properties found for giant gravitons in the Lunin-Maldacena background [24]

${ }^{2}$ We note, however, that the Wilson loop operator (1.1) is half BPS if the curve is taken to be a lightlike line (possible in the Lorentzian case) and with $\dot{y}^{i}=0$. This operator has no coupling to the scalar fields and is not sensitive to the deformation. In this paper we focus in the case where the Wilson loop has coupling to the scalar fields since we are interested in the effects of the $\beta$-deformation. We thanks Nadav Drukker for a discussion on this.

${ }^{3}$ non-BPS in the local sense. For simplicity, unless otherwise stated, we will omit "local" in the following. The meaning should be clear from the context.
} 
Since the 1/4 BPS Wilson loop necessarily involves a non-trivial coupling to the six real scalars field, for the purpose of using AdS/CFT, it is more convenient to re-express the deformed fivesphere metric and the $B$-field in terms of the embedding $\mathbf{R}^{\mathbf{6}}$ coordinates. We then point out some very remarkable properties satisfied by the deformed metric and the $B$-field, which will be needed later. In section 3 we review the argument for the constraint (1.2) on the form of the BPS Wilson loop and show that similar field theory arguments lead to the same form for the Wilson loop operator. We also derive the general form of the modified boundary condition for the dual string in the Lunin-Maldacena background. Finally, we analyze the boundary contribution arising from the Legendre transformation of the action and show that the finiteness of the Wilson loop vev fixes the form of the string boundary condition. We finish, by giving in section 4 the dual string solution in the Lunin-Maldacena background of a near-1/4 BPS circular Wilson loop. Unlike the undeformed case where the string surface is confined on a $S^{2}$ in the five-sphere, the string now extends on a deformed $\tilde{S}^{3}$. The expectation value of the Wilson loop is computed and found to be undeformed. We conjecture the exact expectation value of the Wilson loop is given by the same matrix model as in the undeformed case. A number of appendices are included. In appendix A, we derive the form of the Wilson loop in the large $N$ limit using the phase factor associated with the infinitely massive quark obtained from the breaking $U(N+1) \rightarrow U(N) \times U(1)$. In appendix B, we collect some of the formula of the deformed metric expressed in the Cartesian coordinates. The Hamiltonian-Jacobi equation in the presence of $B$-field is derived in appendix $\mathrm{C}$. In appendix D we show that the 1-loop corrected scalar propagator and gauge boson propagator in the Feynman gauge remains equal. Using this result, we show that our near BPS Wilson loop operator is free from UV divergences up to order $\left(g^{2} N\right)^{2}$.

\section{The Lunin-Maldacena Background}

The type IIB supergravity solution that is dual to the $\beta$-deformation of $\mathcal{N}=4$ super Yang Mills was found in [26]. In the string frame it is:

$$
\begin{aligned}
d s^{2} & =R^{2}\left[d s_{A d S_{5}}^{2}+\sum_{i}\left(d \mu_{i}^{2}+G \mu_{i}^{2} d \phi_{i}^{2}\right)+\hat{\gamma}^{2} G \mu_{1}^{2} \mu_{2}^{2} \mu_{3}^{2}\left(\sum_{i} d \phi_{i}\right)^{2}\right] \\
e^{2 \phi} & =g_{s} G \\
B & =R^{2} \hat{\gamma} G\left(\mu_{1}^{2} \mu_{2}^{2} d \phi_{1} \wedge d \phi_{2}+\mu_{2}^{2} \mu_{3}^{2} d \phi_{2} \wedge d \phi_{3}+\mu_{3}^{2} \mu_{1}^{2} d \phi_{3} \wedge d \phi_{1}\right), \\
C_{2} & =-4 R^{2} \hat{\gamma} \omega_{1} \wedge\left(d \phi_{1}+d \phi_{2}+d \phi_{3}\right), \\
C_{4} & =\omega_{4}+4 R^{4} G \omega_{1} \wedge d \phi_{1} \wedge d \phi_{2} \wedge d \phi_{3},
\end{aligned}
$$

where $R^{4}=4 \pi g_{s} N\left(\right.$ in units where $\left.\alpha^{\prime}=1\right)$,

$$
G^{-1}=1+\hat{\gamma}^{2}\left(\mu_{1}^{2} \mu_{2}^{2}+\mu_{2}^{2} \mu_{3}^{2}+\mu_{3}^{2} \mu_{1}^{2}\right)
$$


The parameter $\hat{\gamma}$ appearing in (2.1) is related to the deformation parameter $\beta$ of the gauge theory by:

$$
\hat{\gamma}=R^{2} \beta
$$

The definition of $\omega_{1}$ and $\omega_{4}$ can be found in [26].

The background has the $U(1)^{3}$ symmetry

$$
\phi_{k} \rightarrow e^{i \delta_{k}} \phi_{k}, \quad \text { for arbitrary constant } \delta_{k}, \quad(k=1,2,3)
$$

This is in correspondence with the $U(1)^{3}$ symmetry (1.6) of the $\beta$-deformed SYM theory.

\subsection{Properties of the deformed metric and $B$-field}

It is convenient to introduce the Cartesian coordinates where the deformed $\tilde{S}^{5}$ is embedded

$$
\begin{array}{ll}
Y^{1}=Y \theta^{1}=Y \mu_{1} \cos \phi_{1}, & Y^{4}=Y \theta^{4}=Y \mu_{1} \sin \phi_{1}, \\
Y^{2}=Y \theta^{2}=Y \mu_{2} \cos \phi_{2}, & Y^{5}=Y \theta^{5}=Y \mu_{2} \sin \phi_{2}, \\
Y^{3}=Y \theta^{3}=Y \mu_{3} \cos \phi_{3}, & Y^{6}=Y \theta^{6}=Y \mu_{3} \sin \phi_{3} .
\end{array}
$$

Here $Y^{2}=\left(Y^{i}\right)^{2}$ and $\left(\theta^{i}\right)^{2}=1$. With respect to this basis, the symmetry (2.4) is translated to

$$
Y_{1}+i Y_{4} \rightarrow e^{i \delta_{1}}\left(Y_{1}+i Y_{4}\right), \quad Y_{2}+i Y_{5} \rightarrow e^{i \delta_{2}}\left(Y_{2}+i Y_{5}\right), \quad Y_{3}+i Y_{6} \rightarrow e^{i \delta_{3}}\left(Y_{3}+i Y_{6}\right) .
$$

The metric (2.1a) becomes

$$
d s^{2}=\frac{R^{2}}{Y^{2}}\left(\sum_{\mu=0}^{3} d X^{\mu} d X^{\mu}+d Y^{2}+Y^{2} d \tilde{\Omega}_{5}^{2}\right)=\frac{R^{2}}{Y^{2}}\left(\sum_{\mu=0}^{3} d X^{\mu} d X^{\mu}+\sum_{i=1}^{6} G_{i j} d Y^{i} d Y^{j}\right)
$$

where $G_{i j}$ is the embedding metric of the deformed $\tilde{S}^{5}$. The diagonal terms of the metric are

$$
G_{i i}=\frac{1}{Y^{2}}\left(\cos ^{2} \phi_{i}+G M_{i} \sin ^{2} \phi_{i}\right), \quad G_{i+3 i+3}=\frac{1}{Y^{2}}\left(\sin ^{2} \phi_{i}+G M_{i} \cos ^{2} \phi_{i}\right), \quad i=1,2,3,
$$

where, for convenience, we have defined the new quantities

$$
M_{1}=1+\hat{\gamma}^{2} \mu_{2}^{2} \mu_{3}^{2}, \quad M_{2}=1+\hat{\gamma}^{2} \mu_{1}^{2} \mu_{3}^{2}, \quad M_{3}=1+\hat{\gamma}^{2} \mu_{1}^{2} \mu_{2}^{2}
$$

The non-diagonal elements are

$$
\begin{aligned}
G_{12} & =\frac{\hat{\gamma}^{2}}{Y^{2}} G \mu_{1} \mu_{2} \mu_{3}^{2} \sin \phi_{1} \sin \phi_{2}, & G_{13} & =\frac{\hat{\gamma}^{2}}{Y^{2}} G \mu_{1} \mu_{2}^{2} \mu_{3} \sin \phi_{1} \sin \phi_{3}, \\
G_{15} & =-\frac{\hat{\gamma}^{2}}{Y^{2}} G \mu_{1} \mu_{2} \mu_{3}^{2} \sin \phi_{1} \cos \phi_{2}, & G_{16} & =-\frac{\hat{\gamma}^{2}}{Y^{2}} G \mu_{1} \mu_{2}^{2} \mu_{3} \sin \phi_{1} \cos \phi_{3}, \\
G_{23} & =\frac{\hat{\gamma}^{2}}{Y^{2}} G \mu_{1}^{2} \mu_{2} \mu_{3} \sin \phi_{2} \sin \phi_{3}, & G_{26} & =-\frac{\hat{\gamma}^{2}}{Y^{2}} G \mu_{1}^{2} \mu_{2} \mu_{3} \sin \phi_{2} \cos \phi_{3} .
\end{aligned}
$$


The elements $G_{45}, G_{46}, G_{24}, G_{34}, G_{56}, G_{35}$ differ respectively from $G_{12}, G_{13}, G_{15}, G_{16}, G_{23}, G_{26}$ by switching all the cos and $\sin$ in each case. The remaining elements are

$$
G_{14}=\frac{1}{2 Y^{2}}\left(1-G M_{1}\right) \sin 2 \phi_{1}, \quad G_{25}=\frac{1}{2 Y^{2}}\left(1-G M_{2}\right) \sin 2 \phi_{2}, \quad G_{36}=\frac{1}{2 Y^{2}}\left(1-G M_{3}\right) \sin 2 \phi_{3} .
$$

In the above we have given the metric elements as a function of the angles. For convenience, we have also recorded in the appendix B the expressions of the metric elements as a function of $Y^{i}$.

Even if as expected this deformed metric is not conformally flat, it displays some remarkable symmetries. One can check that the following identity is satisfied

$$
Y^{i} G_{i j} Y^{j}=1,
$$

which leads to

$$
\theta^{i} g_{i j} \theta^{j}=1
$$

where we have defined

$$
g_{i j}:=Y^{2} G_{i j}
$$

The $g_{i j}$ is finite at the boundary as can be easily seen from (2.8), (2.10), (2.11). Another interesting property of the deformed metric is that

$$
\theta^{i}\left(\partial_{\alpha} g_{i j}\right) \theta^{j}=0
$$

where $\partial_{\alpha}$ is an arbitrary derivative. Also we have

$$
\left(\partial_{\alpha} \theta^{i}\right) g_{i j} \theta^{j}=0
$$

which follows immediately from (2.13), (2.15).

The $B$-field also satisfies an interesting identity. Writing the $B$-field as

$$
B=R^{2} \hat{\gamma} G\left(b_{1}+b_{2}+b_{3}\right),
$$

where

$$
b_{\mathrm{m}}:=\frac{1}{2} \epsilon_{\mathrm{mnk}} \mu_{\mathrm{n}}^{2} \mu_{\mathrm{k}}^{2} d \phi_{\mathrm{n}} \wedge d \phi_{\mathrm{k}}, \quad \mathrm{m}, \mathrm{n}, \mathrm{k}=1,2,3
$$

It is

$$
\begin{aligned}
& b_{3}=Y^{-4}\left(Y^{4} Y^{5} d Y^{1} \wedge d Y^{2}+Y^{1} Y^{2} d Y^{4} \wedge d Y^{5}+Y^{1} Y^{5} d Y^{2} \wedge d Y^{4}-Y^{2} Y^{4} d Y^{1} \wedge d Y^{5}\right), \\
& b_{2}=-Y^{-4}\left(Y^{4} Y^{6} d Y^{1} \wedge d Y^{3}+Y^{1} Y^{3} d Y^{4} \wedge d Y^{6}+Y^{1} Y^{6} d Y^{3} \wedge d Y^{4}-Y^{3} Y^{4} d Y^{1} \wedge d Y^{6}\right), \\
& b_{1}=Y^{-4}\left(Y^{5} Y^{6} d Y^{2} \wedge d Y^{3}+Y^{2} Y^{3} d Y^{5} \wedge d Y^{6}+Y^{2} Y^{6} d Y^{3} \wedge d Y^{5}-Y^{3} Y^{5} d Y^{2} \wedge d Y^{6}\right) .(2.19)
\end{aligned}
$$

It is easy to check that the $B$-field satisfies the following identity

$$
B_{i k} \partial_{\sigma} Y^{k} Y^{i}=0 .
$$


In fact the stronger form

$$
b_{\mathrm{n} i k} \partial_{\sigma} Y^{k} Y^{i}=0
$$

holds for the individual pieces composing the $B$-field.

In our following analysis, we will use the properties (2.13), (2.16), (2.20) of the metric and the $B$-field to study the deformed boundary condition for the macroscopic string ending on the

Wilson loop. It will be interesting to see in which calculation the results (2.21) for the $B$-field will be needed.

\section{Near-BPS Wilson Loop and Twisted Boundary Condition}

\subsection{Form of the Wilson loop operator}

We start out by recalling the arguments for the form of the Wilson loop operator (1.1) and the constraint (1.2) in the original undeformed $\mathcal{N}=4$ case. Firstly, one can examine the unbroken supersymmetry on the Wilson loop operators [27-29]. The Wilson loop operator is locally supersymmetric if the constraint (1.2) is satisfied. A second way is from perturbation theory. One finds that the above constraint must be satisfied in order for the UV-divergence to cancel out in the expectation value of $W$. This is easy to check in the leading order in $g^{2} N:=\lambda$ and can be extended to arbitrary higher orders in $\lambda$ using arguments based on the present $S O(6)$ symmetry [27]. Another way to derive the Wilson loop operator is by decomposing the gauge group $U(N+1) \rightarrow U(N) \times U(1)$ in order to use the W-bosons, that appear from this breaking [7,27]. Finally, the constraint can also be understood from the dual supergravity point of view [27]. Imposing appropriate boundary conditions and then using the Hamilton-Jacobi equation for the minimal surface, one find that only if the constraint (1.2) is satisfied can the minimal surface ends on the boundary of $A d S_{5}$ and giving rises to a finite vev for the Wilson loop. We remark that the first two methods work for any gauge group and any representation, while modifications will be needed in order to generalize the third and the fourth methods to other gauge group or higher representation.

In the $\beta$-deformed theory, as we explained in the introduction, it appears impossible to construct a supersymmetric Wilson loop. On the other hand, supergravity configurations have been constructed whose dual operators would have finite vev. We propose to study this form of the Wilson loop operator (1.1), (1.2) and that it provides the dual of the the D-brane configurations constructed in $[23]^{4}$.

We first give field theory arguments for the choice of this operator in the beta-deformed theories. First, as in the undeformed case, one may define the Wilson loop as the phase factor associated with the $\mathrm{W}$-boson probe arising from the breaking $U(N+1) \rightarrow U(N) \times U(1)$. In appendix A, we calculate the deformed $\mathcal{N}=4$ Lagrangian arising from this decomposition. The action looks quite complicated at finite $N$. However all the $\beta$-dependence drops out in the

\footnotetext{
${ }^{4}$ In this case, the loop is taken to be a circle in the $x$-space and a point in the transverse space $y^{i}$.
} 
large $N$ limit of the classical action and the resulting operator takes the form of (1.1), (1.2). We propose this form of the Wilson loop for any $N$.

Another field theory reason is that if ones tries to derive the constraint in the $\beta$-deformed theory using perturbation methods, the result at the leading order of 't Hooft coupling $\lambda$ is the same as in the undeformed theory since the propagators of the $\beta$-deformed theory are not modified. Hence the UV pole cancels if the condition (1.2) is satisfied, as in the undeformed case. At higher orders of $\lambda$, the $\beta$-deformation breaks the $S O(6)$ invariance of the scalars and the simple argument of the undeformed case does not hold and anymore. However one can check explicitly the gauge boson and scalar propagator remains equal up to order $\lambda$. As a result, the UV divergence cancels out explicitly up to order $\lambda^{2}$ if the constraint (1.2) holds. The details is presented in the appendix D. We conjecture that the UV divergences cancel exactly in the $\beta$ deformed SYM theory. A better understanding of perturbative properties of the beta-deformed theory would give an answer to this problem.

This result is quite remarkable since although the $S O(6)$ symmetry is broken by the $\beta$ deformation, a $S O(6)$ invariant constraint is constructed. The same constraint is also obtained from the SUGRA analysis performed in the next subsections and give support to the validity of this constraint (1.2) and the form (1.1) of the Wilson loop operator.

We next turn to the supergravity picture for support of the form of the constraint (1.2) and the conjecture on the UV finiteness of the Wilson loop. Before we do this, a a comment is in order. In order for the Wilson loop operator to respect the $U(1)^{3}$ symmetry (1.6) of the $\beta$-deformed SYM, one need to assign a corresponding rotation

$$
y_{1}+i y_{4} \rightarrow e^{i \delta_{1}}\left(y_{1}+i y_{4}\right), \quad y_{2}+i y_{5} \rightarrow e^{i \delta_{1}}\left(y_{2}+i y_{5}\right), \quad y_{3}+i y_{6} \rightarrow e^{i \delta_{1}}\left(y_{3}+i y_{6}\right),
$$

to the loop variables $y_{i}$. Here we have used the identification of the scalar fields (A.8). The transformation properties (3.1) and (2.6) leads one to associate $y_{i}$ with $Y_{i}$. This fact is important as, given a specific configuration of the loop variables $y_{i}$ in the field theory, it tells which $Y_{i}$ should be activated for the dual string configuration in supergravity. An example will be shown in section 4 .

\subsection{Deformed boundary conditions}

Since the constraint (1.2) is closely related with the boundary conditions of the dual string we will use it to analyze how these boundary conditions are modified and we will see that they are modified for the directions in the $\tilde{S}^{5}$. We will first derive the most general form of the boundary condition for the string minimal surface. This is given in terms of an arbitrary matrix $\Lambda_{m}^{k}$. Then we show that the field theory constraint is obtained if this matrix is given by the vielbein of the deformed metric. We also show that the UV divergence in the supergravity result is cancelled.

Let $\left(\sigma_{1}, \sigma_{2}\right)=(\tau, \sigma)$ be the worldsheet coordinates ${ }^{5}$. The complex structure $(\alpha, \beta=1,2)$ on the worldsheet

$$
J_{\alpha}^{\beta}=\frac{1}{\sqrt{g}} g_{\alpha \gamma} \epsilon^{\gamma \beta}
$$

\footnotetext{
${ }^{5}$ Note that the conjugate momentum is defined with $\sigma_{2}=\sigma$ taken as the Euclidean time. We have chosen to denote the boundary coordinate $\sigma_{1}$ by $\tau$ so as to conform to the notation in (1.1) which is commonly adopted.
} 
is given in terms of the induced metric $g_{\alpha \beta}$. For the Lunin-Maldacena background, the HamiltonJacobi equation takes the form

$$
G^{i j}\left(P_{i}-i B_{i k} \partial_{1} Y^{k}\right)\left(P_{j}-i B_{j l} \partial_{1} Y^{l}\right)+G^{\mu \nu} P_{\mu} P_{\nu}=G_{i j} \partial_{1} Y^{i} \partial_{1} Y^{j}+G_{\mu \nu} \partial_{1} X^{\mu} \partial_{1} X^{\nu}
$$

where the momentum are

$$
P_{i}=G_{i j} J_{1}^{\beta} \partial_{\beta} Y^{j}+i B_{i k} \partial_{1} Y^{k}, \quad P_{\mu}=G_{\mu \nu} J_{1}^{\beta} \partial_{\beta} X^{\nu} .
$$

The derivation of the HJ equation is given in the appendix. Notice the difference between the undeformed case is that now appears the antisymmetric field $B_{i j}$, which is not zero in the deformed Lagrangian. Furthermore, because we use Euclidean world-sheet, it appears as usual an $i$ in front of the worldsheet coupling to the $B$-field. However, the terms including the antisymmetric field will disappear when we substitute in the Hamilton-Jacobi equation the conjugate momentum and we obtain

$$
g_{i j} J_{1}^{\alpha} J_{1}{ }^{\beta} \partial_{\alpha} Y^{i} \partial_{\beta} Y^{j}+J_{1}^{\alpha}{ }^{\alpha}{ }_{1}^{\beta} \partial_{\alpha} X^{\mu} \partial_{\beta} X^{\mu}=g_{i j} \partial_{1} Y^{i} \partial_{1} Y^{j}+\left(\partial_{1} X^{\mu}\right)^{2},
$$

where we have substituted (2.14) and using that $G_{\mu \nu}=\delta_{\mu \nu} / Y^{2}$.

Now let us determine the boundary conditions for the string coordinates. Suppose that the Wilson loop is parametrized by the values $\left(x^{\mu}\left(\sigma_{1}\right), y^{i}\left(\sigma_{1}\right)\right)$ and choose the world-sheet coordinates such that the boundary is located at $\sigma_{2}=0$. Since the deformation in the dual supergravity background does not appear in the $X^{\mu}$ directions, it is natural to impose the same Dirichlet boundary condition for these coordinates as in the undeformed case:

$$
X^{\mu}\left(\sigma_{1}, 0\right)=x^{\mu}\left(\sigma_{1}\right) .
$$

For the remaining 6 string coordinates $Y^{i}\left(\sigma_{1}, \sigma_{2}\right)$, one can expect the situation to be more complicated since in the Lunin-Maldacena background, the deformations from the standard $A d S$ background occur in these directions. Due to the presence of the $B$-field, the general mixed boundary condition takes the form

$$
J_{1}^{\alpha} \partial_{\alpha} Y^{k}\left(\sigma_{1}, 0\right)+i B_{l}^{k} \partial_{1} Y^{l}\left(\sigma_{1}, 0\right)=\Lambda_{l}^{k} \dot{y}^{l}\left(\sigma_{1}\right)
$$

for some invertible matrix $\Lambda_{l}^{k}$. In addition, for a minimal surface to terminate at the boundary of $A d S_{5}$, we have the Dirichlet conditions $Y^{i}\left(\sigma_{1}, 0\right)=0$, which means

$$
\partial_{1} Y^{i}\left(\sigma_{1}, 0\right)=0
$$

So the above Neumann boundary condition simplifies to

$$
J_{1}^{\alpha} \partial_{\alpha} Y^{k}\left(\sigma_{1}, 0\right)=\Lambda_{l}^{k} \dot{y}^{l}\left(\sigma_{1}\right) .
$$

Inserting the boundary conditions (3.6), (3.8) and (3.9) in the Hamilton-Jacobi equation we find

$$
\dot{x}^{2}-\Lambda_{m}^{k} \Lambda_{n}^{l} g_{k l} \dot{y}^{m} \dot{y}^{n}=\left(J_{1}{ }^{\alpha} \partial_{\alpha} X^{\mu}\right)^{2} .
$$


The term $\left(J_{1}^{\alpha} \partial_{\alpha} X^{\mu}\right)^{2}$ has to be zero near a smooth boundary, otherwise it costs infinite area. Therefore, we arrived at the constraint

$$
\dot{x}^{2}=g_{k l} \Lambda_{m}^{k} \Lambda_{n}^{l} \dot{y}^{m} \dot{y}^{n}
$$

In particular, the constraint derived from supergravity agrees with the constraint (1.2) derived from field theory considerations of the condition if the matrix $\Lambda^{k}{ }_{i}$ satisfies the condition

$$
g_{k l} \Lambda_{m}^{k} \Lambda_{n}^{l}=\delta_{m n}
$$

This means that the boundary condition matrix $\Lambda_{m}^{k}$ is the vielbein of the deformed metric $g_{k l}$. We remark that in [23], the D-brane boundary condition in the $\beta$-deformed theory was obtained out using TsT transformation on the original undeformed boundary condition. It was easy in that case since only angles was involved. In our case we still expect that one can perform a TsTtransformation on the angles to derive the modified boundary condition (3.9), (3.12), although it is less direct since the boundary condition is formulated in terms of the Cartesian coordinates while TsT transformations operates on the angles.

\subsection{Legendre transformation and boundary contribution}

In the undeformed case, after performing a Legendre transformation the UV singularity of the area functional cancels because of the constraint $\dot{x}^{2}=\dot{y}^{2}$. For the deformed case, we should have a similar situation in order for our result to be consistent. We will check this now. As before, since the boundary condition (3.9) is of Neumann type, we consider the same Legendre transform

$$
\tilde{A}=A-\oint d \sigma_{1} P_{i} Y^{i}
$$

Since the metric is singular at $Y=0$, we introduce a regulator $Y=\epsilon$ and evaluate the regularized action for $Y \geq \epsilon$. Let us first focus on the term that comes from Legendre transformation Using the definition (3.4), we have, at $Y=\epsilon$,

$$
P_{i} Y^{i}=G_{i j} J_{1}^{\beta} \partial_{\beta} Y^{j} Y^{i}=\frac{1}{Y} J_{1}^{\alpha} \partial_{\alpha} Y
$$

where we have used the property (2.20) to get rid of the $B$-field term in the first equality; and substituted $Y^{i}=Y \theta^{i}, G_{i j}=g_{i j} / Y^{2}$ and used (2.13), 2.16) in the second equality. To express $J_{1}^{\alpha} \partial_{\alpha} Y$ in terms of the boundary data, we note on substituting $Y^{i}=Y \theta^{i}$ and using again (2.13), (2.16) that,

$$
g_{i j}\left(J_{1}^{\alpha} \partial_{\alpha} Y^{i}\right)\left(J_{1}^{\beta} \partial_{\beta} Y^{j}\right)=\left(J_{1}^{\alpha} \partial_{a} Y\right)^{2}+Y^{2} J_{1}^{\alpha} J_{1}^{\beta} g_{i j} \partial_{\alpha} \theta^{i} \partial_{\beta} \theta^{j} .
$$

In the limit $\epsilon \rightarrow 0$, the second term on the RHS is zero. As for the LHS, we use the boundary condition (3.9) and the condition (3.12). Therefore we obtain

$$
\dot{y}^{2}=\left(J_{1}^{\alpha} \partial_{a} Y\right)^{2} .
$$


And the action (3.13) becomes

$$
\tilde{A}=A-\oint d \sigma_{1} \frac{|\dot{y}|}{Y}=A-\frac{1}{\epsilon} \oint d \sigma_{1}|\dot{y}|,
$$

where we are evaluating the regularized action for $Y \geq \epsilon$.

Now, as in the undeformed case, we expect the area of the minimal surface to have a linear divergence proportional to the circumference of the boundary. Therefore

$$
\tilde{A}=\frac{1}{\epsilon} \oint d \sigma_{1}(|\dot{x}|-|\dot{y}|)+\text { finite part. }
$$

This means that like the undeformed case, the linear divergence in the deformed case cancels when the conditions (1.2) is satisfied.

It is worth noticing that this analysis of the absence of UV divergence in the vev of the Wilson loop is valid for large $\lambda$, while the field theory analysis presented in the last subsection is valid for small (up to second order in) $\lambda$. The fact that the UV divergence cancels and a well-defined Wilson loop is obtained for both small and large $\lambda$ leads us to the conjecture that the Wilson loop (11.1), (11.2) is well-defined and has finite vev in the $\mathcal{N}=1 \beta$-deformed SYM theory.

\section{Near-1/4 BPS Wilson Loop}

In the above, we have proposed that the D-brane configurations considered in [23] are dual to the near-1/2 BPS operators where the circular loop has a trivial dependence in the transverse space. Now we look at next non-trivial case where the loop involves a non-trivial rotation in the transverse space as well,

$$
W[C]=\frac{1}{N} \operatorname{Tr} P \exp \left[\int d \tau\left(i A_{\mu} \dot{x}^{\mu}(\tau)+|\dot{x}(\tau)| \varphi_{i} \theta^{i}(\tau)\right)\right],
$$

where the loop is a circular path of radius $R_{0}$ in space

$$
x^{1}=R_{0} \cos \tau, \quad x^{2}=R_{0} \sin \tau,
$$

and the coupling to the three scalars $\varphi_{1}, \varphi_{2}, \varphi_{5}$ is parametrized by

$$
\theta^{1}=\cos \theta_{0}, \quad \theta^{2}=\sin \theta_{0} \cos \tau, \quad \theta^{5}=\sin \theta_{0} \sin \tau,
$$

with an arbitrary fixed $\theta_{0}$. This operator in the undeformed theory is $1 / 2$ BPS when $\theta_{0}=0$ and $1 / 4$ BPS in general [9]. In this section we use the AdS/CFT correspondence to compute the value for the circular near BPS Wilson loop operator in the $\beta$-deformed SYM.

We use the following form for the (Euclidean) $A d S_{5}$ metric

$$
d s^{2}=d u^{2}+\cosh ^{2} u\left(d \rho^{2}+\sinh ^{2} \rho d \psi^{2}\right)+\sinh ^{2} u\left(d \chi^{2}+\sin ^{2} \chi d \phi^{2}\right) .
$$


For the deformed $\tilde{S}^{5}$ (2.1a), we parametrize the $\mu_{i}$ coordinates via

$$
\mu_{1}=\cos \theta, \quad \mu_{2}=\sin \theta \cos \alpha, \quad \mu_{3}=\sin \theta \sin \alpha
$$

so that $\sum d \mu_{i}^{2}=d \theta^{2}+\sin ^{2} \theta d \alpha^{2}$. For Euclidean space, the worldsheet coupling to the $B$-field get an extra factor of $-i$.

To find the dual string configuration, we note that

$$
\begin{aligned}
& \theta^{1}+i \theta^{4}=\cos \theta_{0}, \\
& \theta^{2}+i \theta^{5}=\sin \theta_{0} e^{i \tau}, \\
& \theta^{3}+i \theta^{6}=0
\end{aligned}
$$

Comparing with the definition (2.5) for $\theta^{i}$, and using (4.5), this means the dual string configuration must satisfy $\phi_{2}=\tau$, and $\theta=\theta_{0}, \phi_{1}=\alpha=\phi_{3}=0$ at the boundary. Minimally, one wants to consider an ansatz involving only two angles $\phi_{2}$ and $\theta$. However due to the $B$-field, one can see easily that this is inconsistent. Let us therefore consider a motion on $R^{2} \times \tilde{S}^{3}$ where $R^{2} \subset A d S_{5}$ is parametrized by $\psi$ and $\rho$, and the deformed 3 -sphere is parametrized by the three angles $\theta, \phi_{1}, \phi_{2}$ with $\alpha=\phi_{3}=0$. The Polyakov action for the Euclidean worldsheet $(\sigma, \tau)$ is

$$
\begin{array}{r}
S=\frac{\sqrt{\lambda}}{4 \pi} \int d \sigma d \tau\left[\rho^{\prime 2}+\dot{\rho}^{2}+\sinh ^{2} \rho\left(\psi^{\prime 2}+\dot{\psi}^{2}\right)+\theta^{\prime 2}+\dot{\theta}^{2}+G \cos ^{2} \theta\left(\phi_{1}^{\prime 2}+\dot{\phi}_{1}^{2}\right)\right. \\
\left.+G \sin ^{2} \theta\left({\phi_{2}^{\prime}}^{2}+\dot{\phi}_{2}^{2}\right)-2 i \hat{\gamma} G \sin ^{2} \theta \cos ^{2} \theta\left(\dot{\phi}_{1} \phi_{2}{ }^{\prime}-\phi_{1}{ }^{\prime} \dot{\phi}_{2}\right)\right]
\end{array}
$$

where $^{\prime}$ (resp. ${ }^{\cdot}$ ) denotes $\partial_{\sigma}$ (resp. $\partial_{\tau}$ ) derivative. Due to the extra factor of $-i$ in the $B$-field coupling, a real configuration is possible only if one perform a Wick rotation $\phi_{1} \rightarrow i \phi_{1}$. To match with the path specified by (4.2), (4.3), we look for solution of the form

$$
\begin{aligned}
& u=0, \quad \rho=\rho(\sigma), \quad \psi=\tau \\
& \theta=\theta(\sigma), \quad \phi_{1}=\phi_{1}(\sigma), \quad \phi_{2}=\tau .
\end{aligned}
$$

We remark that, compared to the solution [30] for the undeformed case, our ansatz has an additional angle $\phi_{1}$ turned on. This is similar to the situation in the story of magnon. There the string configuration dual to the magnon was found [31] to expand from a motion on $S^{2}$ for the undeformed case to a motion on a deformed 3 -sphere when the $\beta$-deformation is turned on. We also remark that the Wick rotation on $\phi_{1}$ is natural and is consistent with a semi-classical interpretation of the AdS/CFT correspondence as a tunnelling phenomena.

The classical equations of motion for our ansatz (4.8), (4.9) takes the form

$$
\begin{aligned}
\rho^{\prime \prime} & =\cosh \rho \sinh \rho \\
\theta^{\prime \prime} & =\frac{1}{2} \partial_{\theta}\left(G \sin ^{2} \theta\right)-\frac{1}{2} \partial_{\theta}\left(G \cos ^{2} \theta\right) \phi_{1}^{\prime 2}+\partial_{\theta}\left(\hat{\gamma} G \sin ^{2} \theta \cos ^{2} \theta\right) \phi_{1}^{\prime} \\
0 & =\partial_{\tau}\left(-G \cos ^{2} \theta \dot{\phi}_{1}-\hat{\gamma} G \sin ^{2} \theta \cos ^{2} \theta \phi_{2}^{\prime}\right)+\partial_{\sigma}\left(-G \cos ^{2} \theta \phi_{1}{ }^{\prime}+\hat{\gamma} G \sin ^{2} \theta \cos ^{2} \theta \dot{\phi}_{2}\right)( \\
0 & =\partial_{\tau}\left(G \sin ^{2} \theta \dot{\phi}_{2}+\hat{\gamma} G \sin ^{2} \theta \cos ^{2} \theta \phi_{1}^{\prime}\right)+\partial_{\sigma}\left(-G \sin ^{2} \theta \phi_{2}{ }^{\prime}-\hat{\gamma} G \sin ^{2} \theta \cos ^{2} \theta \dot{\phi}_{1}\right)
\end{aligned}
$$


The equation (4.13) is satisfied trivially. Equation (4.12) gives

$$
-G \cos ^{2} \theta \phi_{1}{ }^{\prime}+\hat{\gamma} G \sin ^{2} \theta \cos ^{2} \theta=c_{1}
$$

For the surface to be closed, it must be possible to reach $\theta=0$ (north pole) or $\pi$ (south pole), and there the derivatives $\phi_{1}{ }^{\prime}, \phi_{2}{ }^{\prime}$ should be zero since no rotation is possible. Therefore $c_{1}=0$ and we have

$$
\phi_{1}^{\prime}=\hat{\gamma} \sin ^{2} \theta
$$

Equation (4.11) then becomes

$$
\theta^{\prime 2}=\sin ^{2} \theta+c_{2}
$$

where $c_{2}$ is a constant. Notice how the $G$ dependence disappears in the above calculations. Finally, we check also the Virasoro constraints, which reads

$$
\rho^{\prime 2}-\sinh ^{2} \rho+\theta^{\prime 2}-G \sin ^{2} \theta-G \cos ^{2} \theta \phi_{1}^{\prime 2}=0,
$$

which implies

$$
-\rho^{\prime 2}+\sinh ^{2} \rho=\theta^{\prime 2}-\sin ^{2} \theta .
$$

Again here notice that the $G$ dependence disappears. To get a surface in correspondence to a single circle, we set $c_{2}=0$, and the final form of the equations of motion is

$$
\begin{aligned}
& \rho^{\prime 2}=\sinh ^{2} \rho, \\
& \theta^{\prime 2}=\sin ^{2} \theta .
\end{aligned}
$$

This give the solution

$$
\begin{gathered}
\sinh \rho=\frac{1}{\sinh \sigma} \\
\sin \theta=\frac{1}{\cosh \left(\sigma_{0} \pm \sigma\right)} \Leftrightarrow \cos \theta=\tanh \left(\sigma_{0} \pm \sigma\right)
\end{gathered}
$$

and

$$
\phi_{1}=\hat{\gamma}\left(\tanh \left(\sigma \pm \sigma_{0}\right) \mp \tanh \left(\sigma_{0}\right)\right) .
$$

To see how our solution behaves, consider the limits

$$
\begin{aligned}
\sigma \rightarrow 0 & \Rightarrow \rho \rightarrow \infty, \quad \text { and } \quad \theta \rightarrow \theta_{0}, \quad \phi_{1} \rightarrow 0 \\
\sigma \rightarrow \infty & \Rightarrow \rho \rightarrow 0, \quad \text { and } \quad \theta \rightarrow 0 \text { or } \pi
\end{aligned}
$$

Here $\cos \theta_{0}=\tanh \sigma_{0}$. Depending on the sign in (4.21), the surface extends over the north or south pole of $\tilde{S}^{5}$. 
Next we evaluate the action for this configuration. The bulk term is

$$
S_{\text {bulk }}=\frac{\sqrt{\lambda}}{2 \pi} \int d \sigma d \tau\left(\sinh ^{2} \rho+\sin ^{2} \theta\right)
$$

from which we find

$$
S_{\text {bulk }}=\sqrt{\lambda}\left(\operatorname{coth} \rho_{\max } \mp \cos \theta_{0}\right) .
$$

Here we have introduced a cutoff $\sigma_{\min }$ to regulate the boundary contribution, and $\rho_{\max }$ is the corresponding cutoff on $\rho$. The coth $\rho_{\max }$ term will cancel with boundary term coming from the Legendre transformation as we have showed above. Hence, the final result is

$$
S_{t o t}=\mp \sqrt{\lambda} \cos \theta_{0}
$$

and

$$
\langle W\rangle \sim \exp \left( \pm \sqrt{\lambda} \cos \theta_{0}\right),
$$

where the sign is we chosen to minimize the action. This is the same vev as the $1 / 4$ BPS Wilson loop in the undeformed theory.

We note that in addition to this supergravity solution which involves 3 angles, one can also construct a solution which involves only the two angles

$$
\theta=\theta(\sigma), \quad \alpha=\tau,
$$

together with (4.8). This solution is exactly the same as the undeformed one given in [30] and gives rises to the same expectation value for the dual Wilson loop. It is straightforward to work out the Wilson loop operator that is dual to it. It is defined by the loop

$$
\theta_{1}=\cos \theta_{0}, \quad \theta_{2}=\sin \theta_{0} \cos \tau, \quad \theta_{3}=\sin \theta_{0} \sin \tau .
$$

Due to a lack of $S O(6)$ invariance, the Wilson loop operator with the loop (4.30) is different from the one with the loop (4.3). It is quite amazing that they have the same expectation value.

To understand this result better. Let us first recall how the expectation value of the $1 / 2$ BPS circular Wilson loop was computed in gauge theory [33,34]. The circular loop is related to the straight line by a conformal transformation, one can therefore relate the circular Wilson loop to the expectation value of the Wilson straight line, which is one. The result is however nontrivial since under the conformal transformation, the gluon propagator is modified by a singular total derivative which gives non-zero contribution only when both ends of the propagator are located at the point which is conformally mapped to the infinity. It was conjectured by [33] that diagrams with internal vertexes cancel precisely and this is supported by a direct calculation at order $g^{4} N^{2}$. Assuming this is true, [34] showed that the sum of all the non-interacting diagrams can be written as a Hermitian matrix model

$$
\left\langle W_{R}\right\rangle=\left\langle\frac{1}{N} \operatorname{Tr}_{R}\left[e^{M}\right]\right\rangle=\frac{1}{Z} \int \mathcal{D} M \frac{1}{N} \operatorname{Tr}_{R}\left[e^{M}\right] \exp \left(-\frac{2 N}{\lambda} \operatorname{Tr} M^{2}\right) .
$$


This is exact to all order in $\lambda$ and $1 / N$ [34]. Explicit evaluation of the integral and hence the Wilson loop expectation value has been performed for loops in various representations $[9,10,12$, 13,33,34]. This argument has also been applied to the 1/4 BPS fundamental Wilson loop [30].

Now the $\beta$-deformed theory is exact conformal. So the above argument of conformal anomaly applies. The only thing one need to be sure is how interacting diagrams contribute. If they again sum up to zero, then there is no $\beta$-dependence left and one will get the same result as in the undeformed case. Our result of getting the same expectation value for the undeformed and the deformed Wilson loop operators suggests that the interacting diagrams again cancel exactly, at least in the large 't Hooft coupling limit. This is however not easy to prove from perturbation theory since one needs to identify terms with dependence on $\beta^{2} N$ at each order of $1 / N$. We believe a similar mechanism as in the undeformed case is at work. If this is the case, the exact expectation value of the circular Wilson loop in the $\beta$-deformed SYM will be given by the same matrix model as in the undeformed $\mathcal{N}=4$ case. A better understanding of how this works in the undeformed case is necessary and will be very interesting.

For the same reason, we conjecture that the expectation value of the near-1/4 BPS Wilson loop in higher representations will also be unmodified. It will be interesting to construct the D3-brane and D5-brane dual to these Wilson loops in higher representations for the $\beta$-deformed theory and check this.

In this paper we have proposed a definition of a near BPS Wilson loop operator in the $\beta$ deformed SYM theory. We conjectured that this operator has finite vev and provided supporting evidences both from field theory and from supergravity. Thus this operator is a natural candidate of a Wilson loop operator which admits a holographic description in the $\beta$-deformed AdS/CFT correspondence. We showed that on the supergravity side, the finiteness of the vev of the Wilson loop implies the same constraint on the loop as is derived from the field theory analysis. That this is true relies on some remarkable properties satisfied by the metric and the $B$-field of the Lunin-Maldacena background. It will be interesting to be able to formulate and understand these symmetry properties in terms of the dual field theory language. Its origin is likely to be

nonperturbative. This should provide us a better understanding of the mechanism responsible for the finiteness of the vev of the Wilson loop.

\section{Acknowledgements}

We thank Nadav Drukker for useful email exchange and discussions. The research of CSC is supported by EPSRC and PPARC. The work of DG is supported by an EPSRC studentship. 


\section{A. Wilson loop from $U(N+1) \rightarrow U(N) \times U(1)$ breaking}

For real $\beta$-deformation, the bosonic part of the Lagrangian of the $\beta$-deformed SYM theory is given by

$$
\mathcal{L}=\operatorname{Tr}\left(\frac{1}{4} F^{\mu \nu} F_{\mu \nu}+\left(D^{\mu} \bar{\Phi}^{\alpha}\right)\left(D_{\mu} \Phi_{\alpha}\right)-g^{2}\left[\Phi_{\alpha}, \Phi_{\beta}\right]_{*}\left[\bar{\Phi}^{\alpha}, \bar{\Phi}^{\beta}\right]_{*}+\frac{g^{2}}{2}\left[\Phi_{\alpha}, \bar{\Phi}^{\alpha}\right]\left[\Phi_{\beta}, \bar{\Phi}^{\beta}\right]\right)
$$

where $\Phi_{\alpha}(\alpha=1,2,3)$ are the scalar components of the $\mathcal{N}=1$ chiral superfield. The star product for the fields is defined by

$$
f * g:=e^{i \pi \beta\left(Q_{1}^{f} Q_{2}^{g}-Q_{2}^{f} Q_{1}^{g}\right)} f g,
$$

where $f g$ is an ordinary product and $\left(Q_{1}^{\text {field }}, Q_{2}^{\text {field }}\right)$ are the $U(1)_{1} \times U(1)_{2}$ charges of the fields $(f$ or $g)$. The values of the charges for all fields are given in (1.4). Clearly the star product is non-trivial only when different chiral fields are multiplied, as explicit in (A.1). Furthermore, we use the deformed commutator of fields,

$$
\left[f_{\alpha}, g_{\gamma}\right]_{*}:=f_{\alpha} * g_{\gamma}-g_{\gamma} * f_{\alpha}=e^{i \pi \beta_{\alpha \gamma}} f_{\alpha} g_{\gamma}-e^{-i \pi \beta_{\alpha \gamma}} g_{\gamma} f_{\alpha},
$$

where $\beta_{\alpha \gamma}$ takes the values

$$
\beta_{\alpha \gamma}=-\beta_{\gamma \alpha}, \quad \beta_{12}=-\beta_{13}=\beta_{23}:=\beta .
$$

Next, let us break the gauge group $U(N+1) \rightarrow U(N) \times U(1)$ by turning non-zero vacuum expectation values for the scalar fields

$$
\Phi_{\alpha}=\left(\begin{array}{cc}
0_{N \times N} & 0 \\
0 & M \Theta_{\alpha}
\end{array}\right), \quad \alpha=1,2,3 .
$$

Here $\Theta_{\alpha}$ lies on a 5-sphere, $\Theta_{\alpha} \Theta^{\alpha}=1$, corresponding to the direction of the symmetry breaking. Decomposing the fields as

$$
\hat{A}_{\mu}=\left(\begin{array}{cc}
A_{\mu} & W_{\mu} \\
W_{\mu}^{\dagger} & a_{\mu}
\end{array}\right), \quad \hat{\Phi}_{\alpha}=\left(\begin{array}{cc}
\Phi_{\alpha} & W_{\alpha} \\
Y_{\alpha} & M \Theta_{\alpha}
\end{array}\right)
$$


we obtain the action in terms of $W_{\alpha}, Y_{\alpha}$ :

$$
\begin{aligned}
& \hat{S}=\frac{1}{4} F_{\mu \nu}^{2}+\left(D_{\mu} \bar{\Phi}_{\alpha}\right)\left(D_{\mu} \Phi_{\alpha}\right)+\frac{1}{2}\left[\Phi_{\alpha}, \bar{\Phi}_{\alpha}\right]\left[\Phi_{\gamma}, \bar{\Phi}_{\gamma}\right]+\left[\Phi_{\alpha}, \Phi_{\gamma}\right] \beta_{\alpha \gamma}\left[\bar{\Phi}_{\alpha}, \bar{\Phi}_{\gamma}\right] \beta_{\alpha \gamma} \\
& +\left(\left(D_{\mu}-i a_{\mu}\right) W_{\alpha}^{\dagger}\right)\left(\left(D_{\mu}+i a_{\mu}\right) Y_{\alpha}\right)+\left(\left(D_{\mu}+i a_{\mu}\right) Y_{\alpha}^{\dagger}\right)\left(\left(D_{\mu}-i a_{\mu}\right) W_{\alpha}\right) \\
& +\frac{1}{4} f_{\mu \nu}^{2}+\left(\partial_{\mu} M \Theta_{\alpha}^{\dagger}\right)\left(\partial_{\mu} M \Theta_{\alpha}\right) \\
& -2 Y_{\alpha}^{\dagger}\left(\Phi_{\gamma} \Phi_{\alpha} e^{-2 i \pi \beta_{\alpha \gamma}}-\Phi_{\alpha} \Phi_{\gamma}+\frac{1}{2}\left(\Phi_{\alpha}-M \Theta_{\alpha}\right)\left(\Phi_{\gamma}-M \Theta_{\gamma}\right)+M^{2} \Theta_{\alpha} \Theta_{\gamma}\left(e^{2 i \pi \beta_{\alpha \gamma}}-1\right)\right) W_{\gamma}^{\dagger} \\
& -2 Y_{\alpha}\left(\bar{\Phi}_{\gamma} \bar{\Phi}_{\alpha} e^{-2 i \pi \beta_{\alpha \gamma}}-\bar{\Phi}_{\alpha} \bar{\Phi}_{\gamma}+\frac{1}{2}\left(\bar{\Phi}_{\alpha}-M \bar{\Theta}_{\alpha}\right)\left(\bar{\Phi}_{\gamma}-M \bar{\Theta}_{\gamma}\right)+M^{2} \bar{\Theta}_{\alpha} \bar{\Theta}_{\gamma}\left(e^{2 i \pi \beta_{\alpha \gamma}}-1\right)\right) W_{\gamma} \\
& +Y_{\alpha}^{\dagger}\left(\left(2\left(\bar{\Phi}_{\kappa}-M \bar{\Theta}_{\kappa}\right) * *_{\alpha k}\left(\Phi_{\kappa}-M \Theta_{\kappa}\right)+\left[\Phi_{k}, \bar{\Phi}_{\kappa}\right]\right) \delta_{\alpha \gamma}-2\left(\bar{\Phi}_{\gamma}-M \bar{\Theta}_{\gamma}\right) * \beta_{\alpha \gamma}\left(\Phi_{\alpha}-M \Theta_{\alpha}\right)\right. \\
& \left.\quad+\left(\Phi_{\alpha}-M \Theta_{\alpha}\right)\left(\bar{\Phi}_{\gamma}-M \bar{\Theta}_{\gamma}\right)\right) W_{\gamma} \\
& +Y_{\alpha}\left(\left(2\left(\Phi_{\kappa}-M \Theta_{k}\right) * * \beta_{\alpha k}\left(\bar{\Phi}_{\kappa}-M \bar{\Theta}_{\kappa}\right)+\left[\bar{\Phi}_{\kappa}, \Phi_{\kappa}\right]\right) \delta_{\alpha \gamma}-2\left(\Phi_{\gamma}-M \Theta_{\gamma}\right) * \beta_{\alpha \gamma}\left(\bar{\Phi}_{\alpha}-M \bar{\Theta}_{\alpha}\right)\right. \\
& \left.\quad+\left(\bar{\Phi}_{\alpha}-M \bar{\Theta}_{\alpha}\right)\left(\Phi_{\gamma}-M \Theta_{\gamma}\right)\right) W_{\gamma}^{\dagger}+\cdots,
\end{aligned}
$$

where we have defined

$$
\begin{aligned}
& \left(\bar{\Phi}_{\kappa}-M \bar{\Theta}_{\kappa}\right) * *_{\beta_{\alpha \kappa}}\left(\Phi_{\kappa}-M \Theta_{\kappa}\right):=\bar{\Phi}_{\kappa} \Phi_{\kappa}+M^{2} \bar{\Theta}_{\kappa} \Theta_{\kappa}-\bar{\Phi}_{\kappa} M \Theta_{\kappa} e^{2 i \pi \beta_{\alpha \kappa}}-M \bar{\Theta}_{\kappa} \Phi_{\kappa} e^{-2 i \pi \beta_{\alpha \kappa}} \\
& \left(\bar{\Phi}_{\gamma}-M \bar{\Theta}_{\gamma}\right) *_{\beta_{\alpha \gamma}}\left(\Phi_{\alpha}-M \Theta_{\alpha}\right):=\bar{\Phi}_{\gamma} \Phi_{\alpha} e^{2 i \pi \beta_{\alpha \gamma}}+M^{2} \bar{\Theta}_{\gamma} \Theta_{\alpha} e^{-2 i \pi \beta_{\alpha \gamma}}-M \bar{\Theta}_{\gamma} \Phi_{\alpha}-\bar{\Phi}_{\gamma} M \Theta_{\alpha} .
\end{aligned}
$$

In (A.7), $\cdots$ denotes terms of higher order (fourth) in the fields $W, Y$, and $\operatorname{Tr}$ over $U(N)$ is understood.

Next, we go to the real basis by introducing

$$
\begin{aligned}
& \Phi_{1}=\frac{1}{\sqrt{2}}\left(\varphi_{1}+i \varphi_{4}\right), \quad \Phi_{2}=\frac{1}{\sqrt{2}}\left(\varphi_{2}+i \varphi_{5}\right), \quad \Phi_{3}=\frac{1}{\sqrt{2}}\left(\varphi_{3}+i \varphi_{6}\right), \\
& W_{1}=\frac{1}{\sqrt{2}}\left(w_{1}+i w_{4}\right), \quad W_{2}=\frac{1}{\sqrt{2}}\left(w_{2}+i w_{5}\right), \quad W_{3}=\frac{1}{\sqrt{2}}\left(w_{3}+i w_{6}\right)
\end{aligned}
$$

and similarly for $Y_{\alpha}$ and $\Theta_{\alpha}$. The terms $Y_{\alpha}^{\dagger}(\cdots) W_{\gamma}^{\dagger}, Y_{\alpha}(\cdots) W_{\gamma}, Y_{\alpha}(\cdots) W_{\gamma}^{\dagger}$ and $Y_{\alpha}^{\dagger}(\cdots) W_{\gamma}$ become

$$
\begin{aligned}
& \sum_{i=1}^{6} w_{i}^{\dagger}\left[\sum_{j=1}^{6} C_{j j}-C_{i i}^{0}\right] w_{i} \\
& +\sum_{\substack{i j=14,25,36 \\
w_{i}}}^{\dagger}\left[2 \lambda_{i j}-C_{i j}^{0}+2 i \sin 2 \pi \beta \sum_{k l} s_{i k l} \varphi_{k} M \theta_{l}\right] w_{j}+\text { c.c. } \\
& +\sum_{\substack{i j \neq 14,25,36 \\
i \neq j}} w_{i}^{\dagger}\left[2 \Lambda_{i j}-C_{i j}^{0}-2 M^{2} \theta_{i} \theta_{j}(\cos 2 \pi \beta-1)+2 i \sum_{k, l=1}^{6} S_{i k l j} \sin 2 \pi \beta\left(\varphi_{k} \varphi_{l}-M^{2} \theta_{k} \theta_{l}\right)\right] w_{j} \\
& \quad+\text { c.c. }
\end{aligned}
$$


where we have defined

$$
\begin{aligned}
C_{i j} & :=\left(\varphi_{i} e^{i \pi \beta}-M \theta_{i} e^{-i \pi \beta}\right)\left(\varphi_{j} e^{-i \pi \beta}-M \theta_{j} e^{i \pi \beta}\right), \\
\Lambda_{i j} & :=\varphi_{i} \varphi_{j}-\varphi_{j} \varphi_{i} \cos 2 \pi \beta \\
\lambda_{i j} & :=\left[\varphi_{i}, \varphi_{j}\right]
\end{aligned}
$$

and $C_{i j}^{0}=C_{i j}(\beta=0)$. The quantities $s_{i j k}, S_{i j k m}$ are equal to \pm 1 or zero, and their non-zero elements are shown below:

$$
\begin{aligned}
& s_{i k l}=1 \quad \text { for } i k l=125,163,241,236,314,352, \quad \text { and } \quad s_{i k l}=-s_{i l k}, \\
& S_{i k l j}=1 \quad \text { for } i k l j=2451,1245,4512,5124,1643,6431,3164,4316,3562,2356,5623,6235 .
\end{aligned}
$$

We have written our result in this form, so to be clear as much as possible the separation between the deformed and the undeformed part of the Lagrangian.

Following the derivation of [27], one can derive the form of the deformed Wilson loop. What is relevant is the eigenvalues of the mass matrix (A.10). In the undeformed case, the mass matrix has an eigenvalue which is 5-fold degenerated and a zero non-degenerate eigenvalue. The

supersymmetric Wilson loop (1.1), (1.2) is derived from the (infinitely) massive quark probe. In the $\beta$-deformed case, the eigenvalues are generally deformed and degeneracy is lifted. However it is clear that the large $N$ Wilson loop will be the same as in the undeformed case because there isn't any multiplicative factor depending on $N$ in the mass matrix (A.10), therefore the classical Lagrangian is the same as the undeformed one in the large $N$ limit (1.7).

For finite $N$, one will need to keep track of all the dependence of $\beta$ in the Lagrangian (A.10). Due to the large amount of computational work, we were not able to work out the explicit expressions of the eigenvalues. However for the cases we have checked (for example by setting some of the $\phi_{k}$ and $\theta_{k}$ zero), it appears that there is always an eigenvalue which is equal to the undeformed one. It is the phase factor which is associated with this quark which gives rises to the Wilson loop (1.1), (1.2).

We remark that one may also utilize the star product (A.2) and use a star product path ordering to define the Wilson loop operator. Unlike the Wilson loop in the ordinary noncommutative geometry which is highly non-local [35], the closed Wilson loop operator is immediately local and there is no need to employ an open Wilson line. When one expands the exponent, one will get higher and higher powers of the scalar fields and each of them is accompanied with a phase factor which depends on the charge configuration of the scalars. Since these phase factors becomes higher and higher power in $\beta$, in general one cannot drop the $\beta$-dependence even in the large $N$ limit. This operator is not what one obtains from the probe analysis presented above. It is an interesting question whether this noncommutative Wilson loop also admits a nice holographic interpretation, and how.

\section{B. The deformed metric in the Cartesian coordinate system}

For convenience we collect and present the metric in the coordinate system (2.5) expressed in 
$Y^{i}$ coordinates. Defining

$$
\begin{aligned}
& A_{1}=1+\hat{\gamma}^{2} Y^{-4}\left(Y^{2^{2}}+Y^{5^{2}}\right)\left(Y^{3^{2}}+Y^{6^{2}}\right), \\
& A_{2}=1+\hat{\gamma}^{2} Y^{-4}\left(Y^{1^{2}}+Y^{4^{2}}\right)\left(Y^{3^{2}}+Y^{6^{2}}\right), \\
& A_{3}=1+\hat{\gamma}^{2} Y^{-4}\left(Y^{1^{2}}+Y^{4^{2}}\right)\left(Y^{2^{2}}+Y^{5^{2}}\right),
\end{aligned}
$$

the metric elements are:

$$
\begin{array}{ll}
G_{11}=Y^{-2} \frac{\left(Y^{1^{2}}+G Y^{4^{2}} A_{1}\right)}{Y^{1^{2}}+Y^{4^{2}}}, & G_{44}=Y^{-2} \frac{\left(Y^{4^{2}}+G Y^{1^{2}} A_{1}\right)}{Y^{1^{2}}+Y^{4^{2}}}, \\
G_{22}=Y^{-2} \frac{\left(Y^{2^{2}}+G Y^{5^{2}} A_{2}\right)}{Y^{2^{2}}+Y^{5^{2}}}, & G_{55}=Y^{-2} \frac{\left(Y^{5^{2}}+G Y^{2^{2}} A_{2}\right)}{Y^{2^{2}}+Y^{5^{2}}}, \\
G_{33}=Y^{-2} \frac{\left(Y^{3^{2}}+G Y^{6^{2}} A_{3}\right)}{Y^{3^{2}}+Y^{6^{2}}}, & G_{44}=Y^{-2} \frac{\left(Y^{6^{2}}+G Y^{3^{2}} A_{1}\right)}{Y^{3^{2}}+Y^{6^{2}}},
\end{array}
$$

$$
\begin{array}{ll}
G_{12}=2 Y^{-6} \hat{\gamma}^{2} G\left(Y^{3^{2}}+Y^{6^{2}}\right) Y^{4} Y^{5}, & G_{13}=2 Y^{-6} \hat{\gamma}^{2} G\left(Y^{2^{2}}+Y^{5^{2}}\right) Y^{4} Y^{6}, \\
G_{15}=-2 Y^{-6} \hat{\gamma}^{2} G\left(Y^{3^{2}}+Y^{6^{2}}\right) Y^{2} Y^{4}, & G_{16}=-2 Y^{-6} \hat{\gamma}^{2} G\left(Y^{2^{2}}+Y^{5^{2}}\right) Y^{4} Y^{3}, \\
G_{23}=2 Y^{-6} \hat{\gamma}^{2} G\left(Y^{1^{2}}+Y^{4^{2}}\right) Y^{5} Y^{6}, & G_{24}=-2 Y^{-6} \hat{\gamma}^{2} G\left(Y^{3^{2}}+Y^{6^{2}}\right) Y^{1} Y^{5}, \\
G_{26}=-2 Y^{-6} \hat{\gamma}^{2} G\left(Y^{1^{2}}+Y^{4^{2}}\right) Y^{3} Y^{5}, & G_{34}=-2 Y^{-6} \hat{\gamma}^{2} G\left(Y^{2^{2}}+Y^{5^{2}}\right) Y^{1} Y^{6}, \\
G_{35}=-2 Y^{-6} \hat{\gamma}^{2} G\left(Y^{1^{2}}+Y^{4^{2}}\right) Y^{2} Y^{6}, & G_{45}=2 Y^{-6} \hat{\gamma}^{2} G\left(Y^{3^{2}}+Y^{6^{2}}\right) Y^{1} Y^{2}, \\
G_{46}=2 Y^{-6} \hat{\gamma}^{2} G\left(Y^{2^{2}}+Y^{5^{2}}\right) Y^{1} Y^{3}, & G_{56}=2 Y^{-6} \hat{\gamma}^{2} G\left(Y^{1^{2}}+Y^{4^{2}}\right) Y^{2} Y^{3},
\end{array}
$$

$$
\begin{aligned}
& G_{14}=2 Y^{-2} \frac{Y^{1} Y^{4}\left(1-G A_{1}\right)}{Y^{1^{2}}+Y^{4^{2}}}, \\
& G_{25}=2 Y^{-2} \frac{Y^{2} Y^{5}\left(1-G A_{2}\right)}{Y^{2^{2}}+Y^{5^{2}}}, \\
& G_{36}=2 Y^{-2} \frac{Y^{3} Y^{6}\left(1-G A_{3}\right)}{Y^{3^{2}}+Y^{6^{2}}} .
\end{aligned}
$$

Substituting from (2.5) the coordinates we express the metric in angles, the diagonal terms are

$$
\begin{array}{ll}
G_{11}=\frac{1}{Y^{2}}\left(\cos ^{2} \phi_{1}+G \sin ^{2} \phi_{1} M_{1}\right), & G_{44}=\frac{1}{Y^{2}}\left(\sin ^{2} \phi_{1}+G \cos ^{2} \phi_{1} M_{1}\right), \\
G_{22}=\frac{1}{Y^{2}}\left(\cos ^{2} \phi_{2}+G \sin ^{2} \phi_{2} M_{2}\right), & G_{55}=\frac{1}{Y^{2}}\left(\sin ^{2} \phi_{2}+G \cos ^{2} \phi_{2} M_{2}\right), \\
G_{33}=\frac{1}{Y^{2}}\left(\cos ^{2} \phi_{3}+G \sin ^{2} \phi_{3} M_{3}\right), & G_{66}=\frac{1}{Y^{2}}\left(\sin ^{2} \phi_{3}+G \cos ^{2} \phi_{3} M_{3}\right) .
\end{array}
$$


The non-diagonal elements are

$$
\begin{aligned}
& G_{12}=\frac{1}{Y^{2}} \hat{\gamma}^{2} G \mu_{1} \mu_{2} \mu_{3}^{2} \sin \phi_{1} \sin \phi_{2}, \quad G_{13}=\frac{1}{Y^{2}} \hat{\gamma}^{2} G \mu_{1} \mu_{2}^{2} \mu_{3} \sin \phi_{1} \sin \phi_{3}, \\
& G_{15}=-\frac{1}{Y^{2}} \hat{\gamma}^{2} G \mu_{1} \mu_{2} \mu_{3}^{2} \sin \phi_{1} \cos \phi_{2}, \quad G_{16}=-\frac{1}{Y^{2}} \hat{\gamma}^{2} G \mu_{1} \mu_{2}^{2} \mu_{3} \sin \phi_{1} \cos \phi_{3}, \\
& G_{23}=\frac{1}{Y^{2}} \hat{\gamma}^{2} G \mu_{1}^{2} \mu_{2} \mu_{3} \sin \phi_{2} \sin \phi_{3}, \quad G_{24}=-\frac{1}{Y^{2}} \hat{\gamma}^{2} G \mu_{1} \mu_{2} \mu_{3}^{2} \cos \phi_{1} \sin \phi_{2}, \\
& G_{26}=-\frac{1}{Y^{2}} \hat{\gamma}^{2} G \mu_{1}^{2} \mu_{2} \mu_{3} \sin \phi_{2} \cos \phi_{3}, \quad G_{34}=-\frac{1}{Y^{2}} \hat{\gamma}^{2} G \mu_{1} \mu_{2}^{2} \mu_{3} \cos \phi_{1} \sin \phi_{3}, \\
& G_{35}=-\frac{1}{Y^{2}} \hat{\gamma}^{2} G \mu_{1}^{2} \mu_{2} \mu_{3} \cos \phi_{2} \sin \phi_{3}, \quad G_{45}=\frac{1}{Y^{2}} \hat{\gamma}^{2} G \mu_{1} \mu_{2} \mu_{3}^{2} \cos \phi_{1} \cos \phi_{2}, \\
& G_{46}=\frac{1}{Y^{2}} \hat{\gamma}^{2} G \mu_{1} \mu_{2}^{2} \mu_{3} \cos \phi_{1} \cos \phi_{3}, \quad G_{56}=\frac{1}{Y^{2}} \hat{\gamma}^{2} G \mu_{1}^{2} \mu_{2} \mu_{3} \cos \phi_{2} \cos \phi_{3},
\end{aligned}
$$

and

$$
G_{14}=\frac{1}{2 Y^{2}} \sin 2 \phi_{1}\left(1-G M_{1}\right), G_{25}=\frac{1}{2 Y^{2}} \sin 2 \phi_{2}\left(1-G M_{2}\right), G_{36}=\frac{1}{2 Y^{2}} \sin 2 \phi_{3}\left(1-G M_{3}\right) .
$$

\section{Derivation of the Hamilton-Jacobi Equation}

In this appendix we shortly derive the Hamilton-Jacobi (HJ) equation (3.3). Consider the action for the string

$$
S=\int d^{2} \sigma\left(\sqrt{\operatorname{det} g}-i B_{I J} \partial_{1} X^{I} \partial_{2} X^{J}\right)
$$

where $g_{\alpha \beta}:=G_{I J} \partial_{\alpha} X^{I} \partial_{\beta} X^{J}, \alpha, \beta=1,2$. The conjugate momentum is

$$
P_{I}=\frac{\delta S}{\delta\left(\partial_{2} X^{I}\right)}=\frac{1}{\sqrt{g}} G_{I J}\left(g_{11} \partial_{2} X^{J}-g_{12} \partial_{1} X^{J}\right)+i B_{I J} \partial_{1} X^{J}:=\mathcal{P}_{I}+i B_{I J} \partial_{1} X^{J},
$$

where we have introduced $\mathcal{P}_{I}$ as defined above. This turns out to be a convenient variable for expressing the HJ equation. The Hamiltonian is

$$
H=P_{I} \partial_{2} X^{I}-L=\mathcal{P}_{I} \partial_{2} X^{I}-\sqrt{g}
$$

Eliminate $\partial_{2} X^{I}$ in terms of $\mathcal{P}_{I}$ and note that $\mathcal{P}_{I} \partial_{1} X^{I}=0$, we obtain

$$
H=\frac{\sqrt{g}}{g_{11}}\left(G^{I J} \mathcal{P}_{I} \mathcal{P}_{J}-g_{11}\right)
$$

And we obtain the HJ equation $H=0$,

$$
G^{I J} \mathcal{P}_{I} \mathcal{P}_{J}=G_{I J} \partial_{1} X^{I} \partial_{1} X^{J}
$$

This is the form of HJ equation we used in the main text of the paper. 

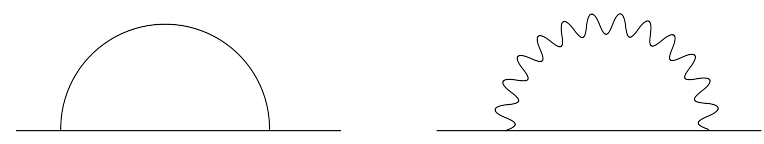

Figure 1: 1-loop contribution to scalar propagator

\section{Cancellation of UV divergences up to order $\left(g^{2} N\right)^{2}$}

We first demonstrate that that the scalar propagator and the gauge boson propagator in the Feynman gauge remains equal up to first order in $g^{2} N$. The simplest way to show this is to use superspace Feynman graphs. In terms of superfields, the Lagrangian for the $\beta$-deformed SYM theory is

$$
\begin{aligned}
L & =\int d^{2} \theta d^{2} \bar{\theta} \operatorname{Tr}\left(e^{-g V} \bar{\Phi}_{i} e^{g V} \Phi_{i}\right)+\frac{1}{2 g^{2}} \int d^{2} \theta \operatorname{Tr} W^{\alpha} W_{\alpha}+\text { c.c. } \\
& +i h \int d^{2} \theta \operatorname{Tr}\left(e^{i \pi \beta} \Phi_{1} \Phi_{2} \Phi_{3}-e^{-i \pi \beta} \Phi_{1} \Phi_{3} \Phi_{2}\right)+i h^{*} \int d^{2} \bar{\theta} \operatorname{Tr}\left(e^{i \pi \beta} \bar{\Phi}_{1} \bar{\Phi}_{2} \bar{\Phi}_{3}-e^{-i \pi \beta} \bar{\Phi}_{1} \bar{\Phi}_{3} \bar{\Phi}_{2}\right) .
\end{aligned}
$$

Using $f_{a b c}:=-i \operatorname{Tr}\left(T_{a}\left[T_{b}, T_{c}\right]\right), \quad d_{a b c}:=\operatorname{Tr}\left(T_{a}\left\{T_{b}, T_{c}\right\}\right)$, the superpotential can be written as

$$
-h\left(f_{a b c} \cos \pi \beta+d_{a b c} \sin \pi \beta\right) \int d^{2} \theta \Phi_{1}^{a} \Phi_{2}^{b} \Phi_{3}^{c}+\text { c.c. . }
$$

The relation between $h$ and $g$ is obtained from the requirement of superconformal invariance, which gives up to two-loop order $[36,37]$,

$$
|h|^{2}\left(C_{2} \cos ^{2} \pi \beta+D_{2} \sin ^{2} \pi \beta\right)=N g^{2} .
$$

Here $f_{a b c} f_{a^{\prime} b c}=\delta_{a a^{\prime}} C_{2}, d_{a b c} d_{a^{\prime} b c}=\delta_{a a^{\prime}} D_{2}$ and $\operatorname{Tr}\left(T_{a} T_{a^{\prime}}\right)=\delta_{a a^{\prime}} / 2$. Now the 1-loop correction to the scalar propagator is contained in the diagrams in figure 1 . It is obvious that the graph (b) is independent of $\beta$. For the graph (a), it has a interaction vertex proportional to $|h|^{2}\left(f_{a b c} f_{a^{\prime} b c} \cos ^{2} \pi \beta+d_{a b c} d_{a^{\prime} b c} \sin ^{2} \pi \beta\right)$. Using the superconformal invariance condition (D.3), this is equal to $g^{2} N \delta_{a a^{\prime}}$ and is independent of $\beta$. Thus the one loop contribution to the scalar propagator is independent of $\beta$. It is obvious that the one loop contribution to the gauge boson propagator is also independent of $\beta$. Using the result of [33], we conclude that the scalar propagator and the gauge boson propagator remains equal up to first order in $g^{2} N$.

Using this result, it is easy to see that the Wilson loop operator (1.1) is free from UV divergence up to order $\left(g^{2} N\right)^{2}$ if the constraint (1.2) is satisfied. The proof is a slight adaption of the computation of [29]. At leading and next-to-leading orders, we have the Feynman diagrams given in figure 2. The linear divergences in diagrams (a-g) got cancelled out immediately due to

the equality of the 1-loop corrected scalar and gauge boson propagators. As for the diagrams (h) and (i), we have

$$
\begin{aligned}
(h)+(i)=2\left(g^{2} N\right)^{2} \int d^{4} x \oint d s_{1} d s_{2} d s_{3} \theta_{c}\left(s_{1}, s_{2}, s_{3}\right) . \\
\qquad \cdot\left(D_{x x_{1}} \partial_{\lambda} D_{x x_{2}}-\partial_{\lambda} D_{x x_{1}} D_{x x_{2}}\right) D_{x x_{3}} \dot{x}_{3}^{\lambda} \cdot\left(\dot{x}_{1}^{\mu} \dot{x}_{2}^{\nu} \delta_{\mu \nu}-\dot{y}_{1}^{i} \dot{y}_{2}^{j} \delta_{i j}\right) .
\end{aligned}
$$



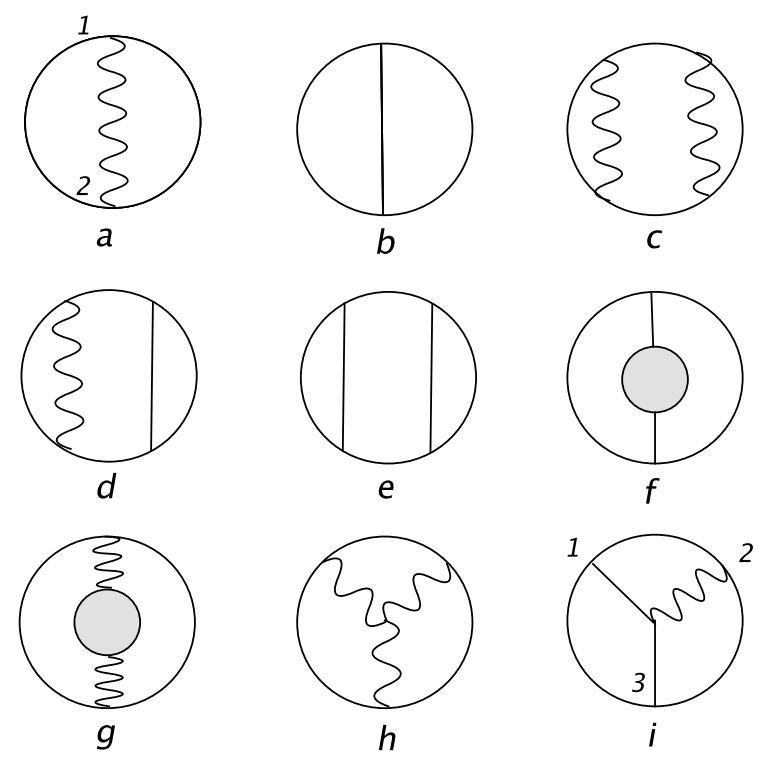

Figure 2: Feynman diagrams of leading and next-to-leading orders

The contribution to (D.4) from the region $s_{1} \sim s_{2} \sim s_{3}$ is linear divergent for a generic loop,

$$
(h)+(i) \sim \oint d s_{1} \frac{1}{\epsilon}\left(\dot{x}_{1}^{2}-\dot{y}_{1}^{2}+\epsilon\right) .
$$

However when the constraint (1.2) is satisfied, the contribution is finite. Thus we conclude that the Wilson loop operator (1.1) has a expectation value that is free from UV divergence up to order $\left(g^{2} N\right)^{2}$ when the constraint is satisfied.

We also remark that, due to the equality of the propagators, the Wilson loop operator with the constraint [29]

$$
\dot{y}^{i}=M_{\mu}^{i} \dot{x}^{\mu}, \quad M_{\mu}^{i} M_{\nu}^{i}=\delta_{\mu \nu}
$$

has expectation value 1 up to order $\left(g^{2} N\right)^{2}$. We conjecture that this Wilson loop has an exact expectation value 1 just as in the $\mathcal{N}=4$ theory.

\section{References}

[1] J. M. Maldacena, "The large N limit of superconformal field theories and supergravity," Adv. Theor. Math. Phys. 2 (1998) 231 [Int. J. Theor. Phys. 38 (1999) 1113] hep-th/9711200.

[2] S. S. Gubser, I. R. Klebanov and A. M. Polyakov, Phys. Lett. B 428 (1998) 105 [arXiv:hep-th/9802109].

[3] E. Witten, Adv. Theor. Math. Phys. 2 (1998) 253 [arXiv:hep-th/9802150].

[4] O. Aharony, S. S. Gubser, J. M. Maldacena, H. Ooguri and Y. Oz, Phys. Rept. 323 (2000) 183 [arXiv:hep-th/9905111]. 
[5] S. Corley, A. Jevicki and S. Ramgoolam, "Exact correlators of giant gravitons from dual N =4 SYM theory," Adv. Theor. Math. Phys. 5 (2002) 809 [arXiv:hep-th/0111222].

[6] D. Berenstein, "A toy model for the AdS/CFT correspondence," JHEP 0407 (2004) 018 [arXiv:hep-th/0403110].

[7] J. M. Maldacena, "Wilson loops in large N field theories," Phys. Rev. Lett. 80 (1998) 4859 [arXiv:hep-th/9803002].

[8] S. J. Rey and J. T. Yee, "Macroscopic strings as heavy quarks in large N gauge theory and anti-de Sitter supergravity," Eur. Phys. J. C 22 (2001) 379 [arXiv:hep-th/9803001].

[9] N. Drukker and B. Fiol, "All-genus calculation of Wilson loops using D-branes," JHEP 0502 (2005) 010 [arXiv:hep-th/0501109].

[10] S. Yamaguchi, "Wilson loops of anti-symmetric representation and D5-branes," JHEP 0605 (2006) 037 [arXiv:hep-th/0603208].

[11] J. Gomis and F. Passerini, "Holographic Wilson loops," JHEP 0608 (2006) 074 [arXiv:hep-th/0604007].

[12] K. Okuyama and G. W. Semenoff, "Wilson loops in N = 4 SYM and fermion droplets," JHEP 0606 (2006) 057 [arXiv:hep-th/0604209].

[13] S. A. Hartnoll and S. P. Kumar, "Higher rank Wilson loops from a matrix model," JHEP 0608 (2006) 026 [arXiv:hep-th/0605027].

[14] J. Gomis and F. Passerini, "Wilson loops as D3-branes," JHEP 0701 (2007) 097 [arXiv:hep-th/0612022].

[15] S. A. Hartnoll and S. Prem Kumar, "Multiply wound Polyakov loops at strong coupling," Phys. Rev. D 74 (2006) 026001 [arXiv:hep-th/0603190].

[16] N. Drukker, S. Giombi, R. Ricci and D. Trancanelli, "On the D3-brane description of some 1/4 BPS Wilson loops," JHEP 0704 (2007) 008 [arXiv:hep-th/0612168].

[17] H. Lin, O. Lunin and J. M. Maldacena, "Bubbling AdS space and 1/2 BPS geometries," JHEP 0410 (2004) 025 [arXiv:hep-th/0409174].

[18] S. Yamaguchi, "Bubbling geometries for half BPS Wilson lines," Int. J. Mod. Phys. A 22 (2007) 1353 [arXiv:hep-th/0601089].

[19] O. Lunin, "On gravitational description of Wilson lines," JHEP 0606 (2006) 026 [arXiv:hep-th/0604133].

[20] J. Gomis and C. Romelsberger, "Bubbling defect CFT's," JHEP 0608 (2006) 050 [arXiv:hep-th/0604155].

[21] E. D'Hoker, J. Estes and M. Gutperle, "Gravity duals of half-BPS Wilson loops," JHEP 0706 (2007) 063 [arXiv:0705.1004 [hep-th]]. 
[22] R. Hernandez, K. Sfetsos and D. Zoakos, "Gravity duals for the Coulomb branch of marginally deformed N = 4 Yang-Mills," JHEP 0603 (2006) 069 [arXiv:hep-th/0510132].

[23] E. Imeroni and A. Naqvi, "Giants and loops in beta-deformed theories," JHEP 0703 (2007) 034 [arXiv:hep-th/0612032].

[24] M. Pirrone, "Giants on deformed backgrounds," JHEP 0612 (2006) 064 [arXiv:hep-th/0609173].

[25] D. Berenstein, J. M. Maldacena and H. Nastase, "Strings in flat space and pp waves from N = 4 super Yang Mills," JHEP 0204 (2002) 013 [arXiv:hep-th/0202021].

[26] O. Lunin and J. Maldacena, "Deforming field theories with U(1) x U(1) global symmetry and their gravity dual," JHEP 0505 (2005) 033 [arXiv:hep-th/0502086].

[27] N. Drukker, D. J. Gross and H. Ooguri, "Wilson loops and minimal surfaces," Phys. Rev. D 60 (1999) 125006 [arXiv:hep-th/9904191].

[28] M. Bianchi, M. B. Green and S. Kovacs, "Instanton corrections to circular Wilson loops in N = 4 supersymmetric Yang-Mills," JHEP 0204 (2002) 040 [arXiv:hep-th/0202003].

[29] K. Zarembo, "Supersymmetric Wilson loops," Nucl. Phys. B 643 (2002) 157 [arXiv:hep-th/0205160].

[30] N. Drukker, '1/4 BPS circular loops, unstable world-sheet instantons and the matrix model," JHEP 0609 (2006) 004 [arXiv:hep-th/0605151].

[31] C. S. Chu, G. Georgiou and V. V. Khoze, "Magnons, classical strings and beta-deformations," JHEP 0611 (2006) 093 [arXiv:hep-th/0606220].

[32] A. Miwa and T. Yoneya, "Holography of Wilson-loop expectation values with local operator insertions," JHEP 0612 (2006) 060 [arXiv:hep-th/0609007].

[33] J. K. Erickson, G. W. Semenoff and K. Zarembo, "Wilson loops in N = 4 supersymmetric Yang-Mills theory," Nucl. Phys. B 582 (2000) 155 [arXiv:hep-th/0003055].

[34] N. Drukker and D. J. Gross, "An exact prediction of N = 4 SUSYM theory for string theory," J. Math. Phys. 42 (2001) 2896 [arXiv:hep-th/0010274].

[35] N. Ishibashi, S. Iso, H. Kawai and Y. Kitazawa, "Wilson loops in noncommutative Yang-Mills," Nucl. Phys. B 573 (2000) 573 [arXiv:hep-th/9910004].

[36] D. Z. Freedman and U. Gursoy, "Comments on the beta-deformed N = 4 SYM theory," JHEP 0511 (2005) 042 [arXiv:hep-th/0506128].

[37] S. Penati, A. Santambrogio and D. Zanon, "Two-point correlators in the beta-deformed N = 4 SYM at the next-to-leading order," JHEP 0510 (2005) 023 [arXiv:hep-th/0506150]. 